\title{
Differential Diagnosis of Malignant Melanoma and Benign Cutaneous Lesions by Ultrasound Analysis
}

\author{
M. Hambardzumyan ${ }^{a^{*}}$, A. Hayrapetyan ${ }^{\text {b }}$ \\ ${ }^{a}$ Derma Clinic Dr. med. Philippe Manuel, Rheinstetten, Germany. \\ ${ }^{b}$ Endovision Medical Center, Yerevan, Armenia.
}

Received 14 March 2020; Accepted 11 May 2020

\begin{abstract}
Background: The purpose of the study is to evaluate the assessment of ultrasound analysis in the differential diagnosis of skin melanoma and benign cutaneous lesions. Objective: 61 patients ( 23 men and 38 women) between 17 and 87 years of age, with melanomas, atheromas, hemangiomas, keratoses, and naevi were studied. Methods: High-frequency gray-scale ultrasound analysis, color Doppler, power Doppler, advanced dynamic flow, strain Elastography, digital Dermoscopy were performed in all cases. Results: In malignant melanoma cases we have mainly: sharp margins, hypoechoic, homogenous structure, absent of posterior shadowing, central and disorganized circulatory pattern with multiple peduncles. In some benign pathology, several ultrasound criteria were exclusive: microcalcifications are only in atheroma, posterior shadowing, and circular rim - in keratosis. The incidence of other ultrasound criteria can vary in atheroma, hemangioma, keratosis, and nevus. Tumor longitudinal and thickness relation were higher (7.9 \pm 1.96$)$ than in all benign pathologies (2.1-4.8). The Elastography stiffness of the 26 skin melanomas was $2.95 \pm 0.18$ and was higher than the group of 35 patients with all benign skin pathology $(0.96 \pm 0.59)$, including atheroma $(2.0 \pm 0.78)$, hemangioma $(0.55 \pm 0.21)$, keratosis $(1.21 \pm 0.21)$ and nevus $(0.78 \pm 0.45)$. Conclusion: Multimodal approaches to exploring highfrequency ultrasound analytic criteria can be helpful in the differential diagnosis of malignant melanoma and benign cutaneous lesions.
\end{abstract}

Keywords: Differential Diagnosis; Melanoma; Atheroma; Hemangioma; Keratosis; Nevus; Dermoscopy; Ultrasound Criteria; Color Doppler (CD); Power Doppler (PD); Advanced Dynamic Flow (ADF); Longitudinal and Thickness (L/T) Relation; Elastography.

\section{Introduction}

Skin pathologies are mostly visible pathologies, therefore, the main efforts were focused on the different optical visualization methods, intended to improve the diagnostic accuracy of melanomas. In the early detection of malignant melanoma during the last 25 years the key factor the mnemonic "ABCDE" to facilitate different visual changes [1]. Standard and advanced photographic methods magnify and/or enhance malignant features of individual melanocytic lesions like confocal microscopy [2], in total, 59,858 subjects were screened in 20 countries and Dermoscopy was used in $78 \%$ of examinations with clinically suspected melanoma [3].

Despite to its objective privileges of improving the optical visualizations, some doctors try to implement the radiological methods in dermatology. The first publication with "skin" and "ultrasound" phrases to be found through the PubMed search engine is the about Doppler ultrasonic detector measuring methods as the valuable help in dermatological angiology for the assessment of vascular problems by Z. Hautkr [4].

\footnotetext{
* Corresponding author: hambardzumyan.michael@gmail.com

doi) http://dx.doi.org/10.28991/SciMedJ-2020-0202-7

$>$ This is an open access article under the CC-BY license (https://creativecommons.org/licenses/by/4.0/).

(C) Authors retain all copyrights.
} 
Later in 1986 W.Strasser et al. [5] reported about B-scan ultrasound in dermatological practice. There are publication reviews analysis the most common dermatologic applications of sonography with gray-scale ultrasound, $\mathrm{CD}$ in some technical considerations for performing this type of examination in normal skin, common benign pathologies including cysts, vascular, infection, inflammatory diseases and malignant pathologies, including basal cell carcinomas and melanomas [6]. Doppler sonography with d-galactose-based signal enhancement permitted the better differential diagnosis of skin 48 malignant and 33 benign tumors than unenhanced Doppler sonography [7]. Tumor thickness is of great importance in the management of cutaneous malignant melanoma because it is used to determine the prognosis and is a key factor in planning surgical margins and selecting candidates for sentinel node biopsy [8]. The accuracy of cutaneous ultrasound as a tool for measuring the tumor thickness analyzed in a consecutive series of 79 primary melanomas [9]. The sensitivity, specificity, positive and negative predictive values for diagnosis of thick melanomas (>1 mm) were $82 \%, 80 \%, 54 \%$, and $94 \%$ respectively. These results suggest that cutaneous ultrasound would properly stage a tumor and allow a single surgical intervention to be planned in approximately $80 \%$ of melanomas. However, it would not provide an accurate measurement on which to base a therapeutic decision in $20 \%$ of melanomas.

High-frequency ultrasound shows promise for the detection of certain changes in the skin and this has implications for early detection of changes associated with pressure ulcer formation and wound healing [10]. They demonstrated the utility of using gray-scale ultrasound to obtain noninvasive, objective, quantitative measurements of wound healing instead of relying only on visual inspection.

The combination of Doppler ultrasound and strain Elastography demonstrates that the assessment of tumor vascularization and stiffness in skin melanomas may contribute to a complex diagnosis before surgical excision [1114]. Both Doppler ultrasound and strain Elastography may provide useful information for the management of cutaneous melanoma patients.

The aim of the study is to evaluate the different ultrasound criteria for the differential diagnosis of skin melanoma and non-melanoma skin benign pathologies.

\section{Materials and Methods}

In this research, we analyzed 61 patients (23 men and 38 women) prospectively admitted to the Endovision Medical Center and National Center of Oncology between 2015 and 2017. The age range was from 17 to 87 years, and the mean age was 48.5 years. Each patient underwent a clinical examination followed by dermoscopy with conventional and polarized lights. Furthermore, we used high-frequency gray-scale ultrasound, Doppler ultrasound methods, including Color Doppler (CD) for revealing blood slow flow, Power Doppler (PD) and advances dynamic flow (ADF). Strain wave Elastography was used to reveal the stiffness of melanoma and benign skin pathologies. All 26 examined patients with primary cutaneous melanoma and the group of 35 patients with benign skin pathology (17 naevi, 7 keratoses, 7 hemangiomas, and 4 atheromas) underwent surgery. Clark and Breslow's classifications were included in the pathological diagnosis of melanoma, as well was performed histological examination using hematoxylin and eosin staining.

\subsection{Equipment}

High-resolution gray-scale ultrasound images followed CD, PD, ADF, Strain Elastography were obtained using Toshiba Xario TUS-X200 (Toshiba Medical System, Tokyo, Japan). We used $8 \mathrm{MHz}$ probes with the extended field of view, zooming and panoramic reconstruction software improving information quality. In 17 cases we used "Aquaflex" ultrasound Gel Pad, 2 cm thickness (Parker Laboratories Inc., Fairfield NJ 07004, USA). Dermoscopic images were captured using DermLite DL3N Dermatoscope (3Gen Inc., San Juan Capistrano, CA, USA).

\subsection{Ultrasound}

Ultrasound analysis can predict the dermal mass nature before cytological or pathological examinations doing before and after surgery [15]. That is why we decided to use some gray-scale ultrasound analytic criteria [16] that were successfully performed for many years for breast pathologies. Gray-scale ultrasound analytic criteria for the skin masses included: margin analysis, internal echo pattern, echo genesis, retro mass acoustic pattern, and the relation between two dimensions. In skin malignant or benign changes, ultrasound usually presents as hypoechoic areas in contrast to the adjacent healthy tissue $[17,18]$. As the cutaneous lesions may be asymmetric, the measurement of the tumor thickness should be based on the site of greater invasion $[18,19]$.

In 17 cases it was more beneficial to use Standoff Gel Pad providing better contact with uneven skin pathological surface. The last technique can be very appropriate and practical also for studying benign skin pathology especially with an irregular surface or by multiple lesions located in a small area. In each, case the tumor thickness (T) was 
measured, together with length (L) thereafter the ratio between $\mathrm{L}$ and $\mathrm{T}$ : $\mathrm{L} / \mathrm{T}$ relation. We checked the masses for additional ultrasound criteria such as the acoustical rim.

Doppler examination, including CD, PD, and ADF can define the mass vascularization, a number of its peduncles, Doppler circulatory pattern, and Doppler vessel distribution. Strain wave Elastography gives us quantified stiffness of the dermal masses.

\subsection{Statistical Methods}

Statistical calculations were done using Graph Pad Prism 6 software. T-test and One Way ANOVA were used for statistical analysis, whereas the T-test was used in nominal variables with 2 groups only and One Way ANOVA in nominal variables with more than 2 groups. Graphics were built by the same software.

\section{Results}

Analyzing the ultrasound criteria in 26 patients with malignant melanoma, we registered a sharp margin with oval, jagged or linear shape (Table 1). Hypoechoic echogenicity and homogenous internal echo pattern were common for melanoma, thought the retro masses acoustic enhancements absent in 14 and registered in 12 cases. The mean number of melanoma lesions length and thickness ratio L/T was $7.9 \pm 1.96$ and it was higher than all benign pathologies, (2.14.8). Doppler mass analysis has mainly central, disorganized, hypervascularization, with multiple peduncles. The Elastography stiffness of 26 skin melanoma patients was higher $(2.95 \pm 0.18)$ the group of 35 patients (Figure 1) with all benign skin pathology $(0.96 \pm 0.59, \mathrm{P}<0.0001)$.

Table 1. Ultrasound analysis of malignant melanoma and skin benign pathologies ( $\mathrm{N}$ of cases/ $\mathrm{N}$ of Group)

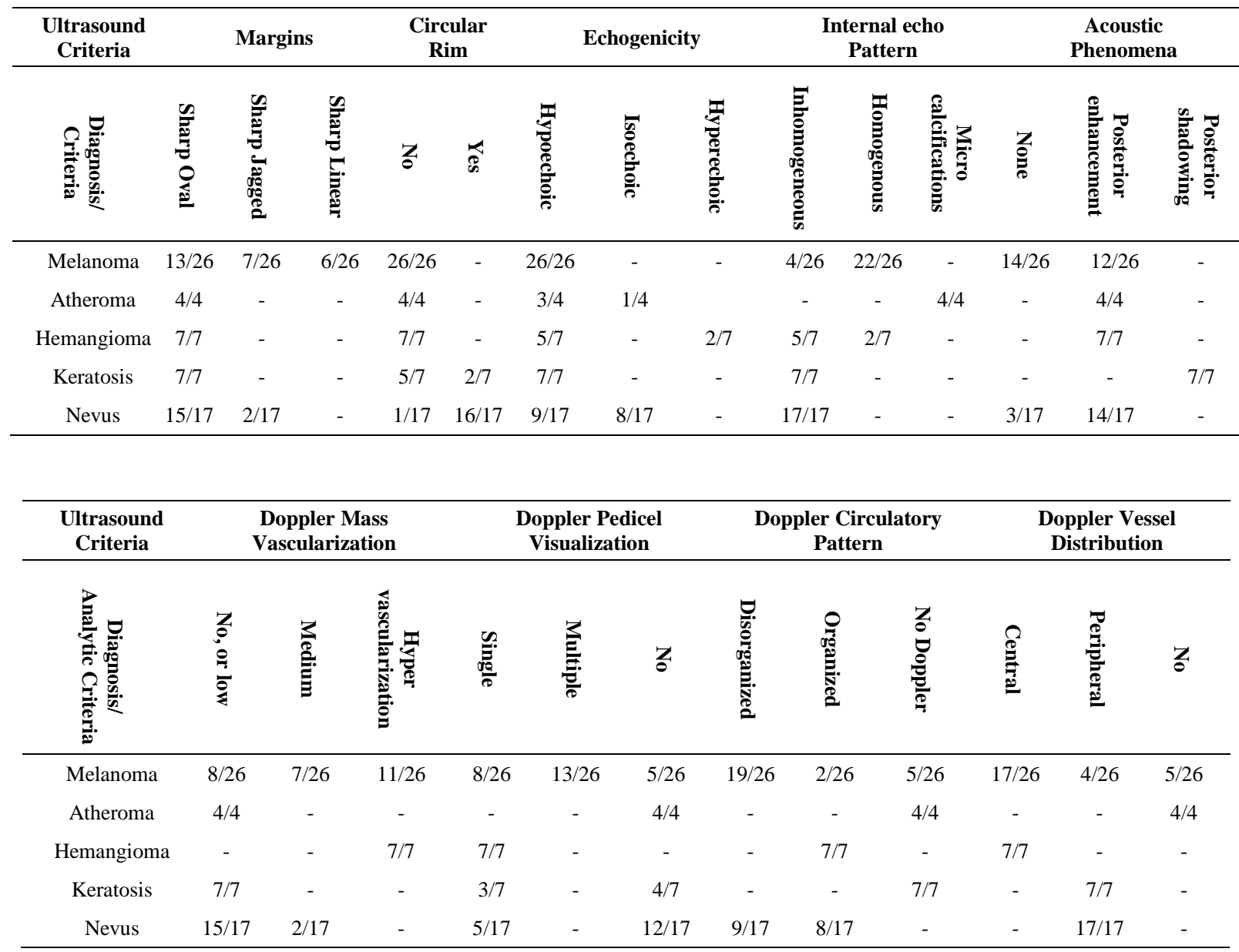




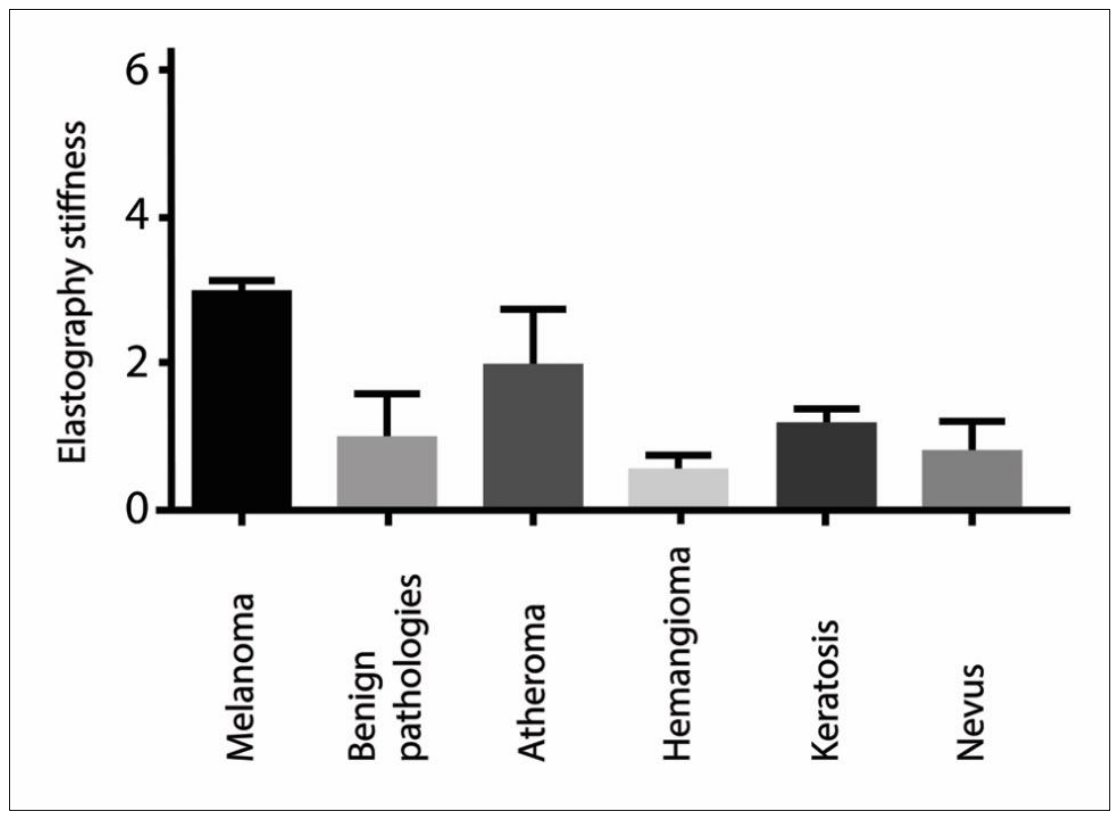

Figure 1. Elastography stiffness of skin melanoma, patients with benign skin pathology, including atheroma, hemangioma, keratosis and nevus

For atheroma, as a benign pathology is a characteristic sharp and smooth margin with absent of a circular rim, hypoechoic (mainly few echoic calcifications) (Figure 2), retro mass acoustic phenomena showing posterior shadowing and less enhancement (Table 1) and L/T relations is $2.15 \pm 0.63$. Doppler mass analysis showed absent of Doppler flow. Elastography stiffness was $2.0 \pm 0.78, \mathrm{P}<0.5$.

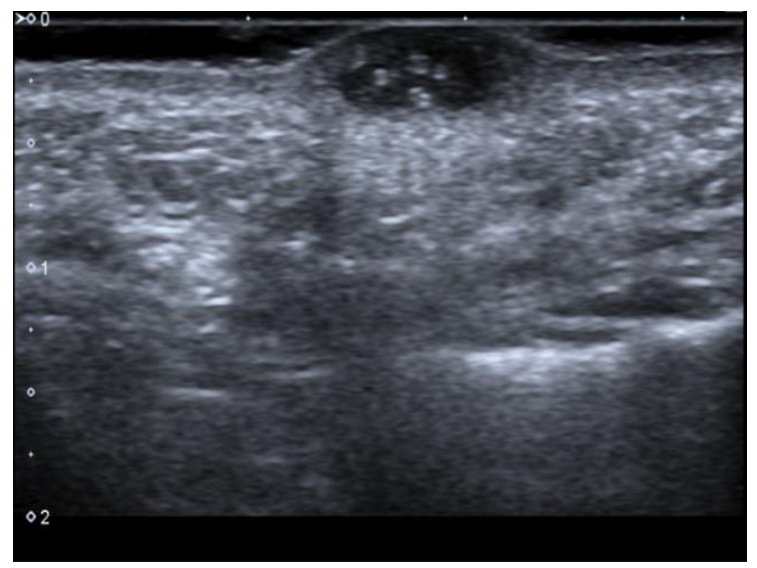

Figure 2. Gray scale ultrasound of atheroma with oval sharp, smooth margin and micro calcifications internal echo pattern, also posterior acoustic enhancement
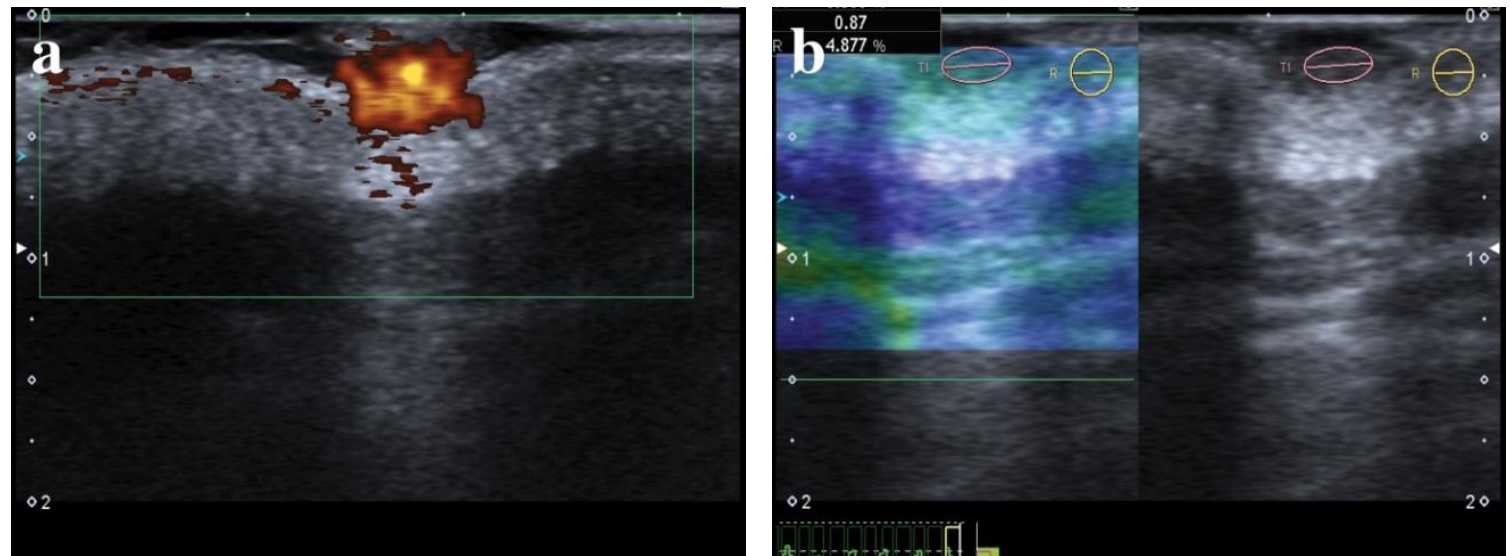

Figure 3. Power Doppler and of hemangioma showing hyper vascularization of the mass (a) also noted in melanoma, in the meantime the Elastography (b) stiffness ratio is much lower - 0.87 
Hemangioma has a sharp and smooth margin (Table 1). Doppler mass analysis finds central, organized, hypervascularization, with single peduncles (Figure 3). Ultrasound characteristics also include absents of the circular rim, hypoechoic or hyperechoic mainly inhomogeneous, with posterior enhancement (Figure 4b) as retro mass acoustic phenomena and L/T relation was $4.17 \pm 2.63$. Elastography stiffness was $0.55 \pm 0.21, \mathrm{P}<0.05$. Hemangioma usually varies in its echogenicity and vascularization according to its phase [6]. In the proliferative early phase, it tends to be hypoechoic and hypervascular. Later, during the partial regression phase, the echogenicity turns heterogeneous, usually presenting a mix of hypoechoic-hyper vascular and hyperechoic-hypo vascular areas.

Sharp and smooth margins are more common for keratosis (Table 1), with some evidence of circular rim, hyperechoic mainly inhomogeneous echogenicity, and posterior shadow (Figure 4b) as the retro mass acoustic phenomena and L/T relation was 4.76 \pm 2.29 . Doppler mass analysis showed absent of flow sometimes single peduncles could be visualized. Elastography stiffness ratio was $1.21 \pm 0.21, \mathrm{P}<0.5$.
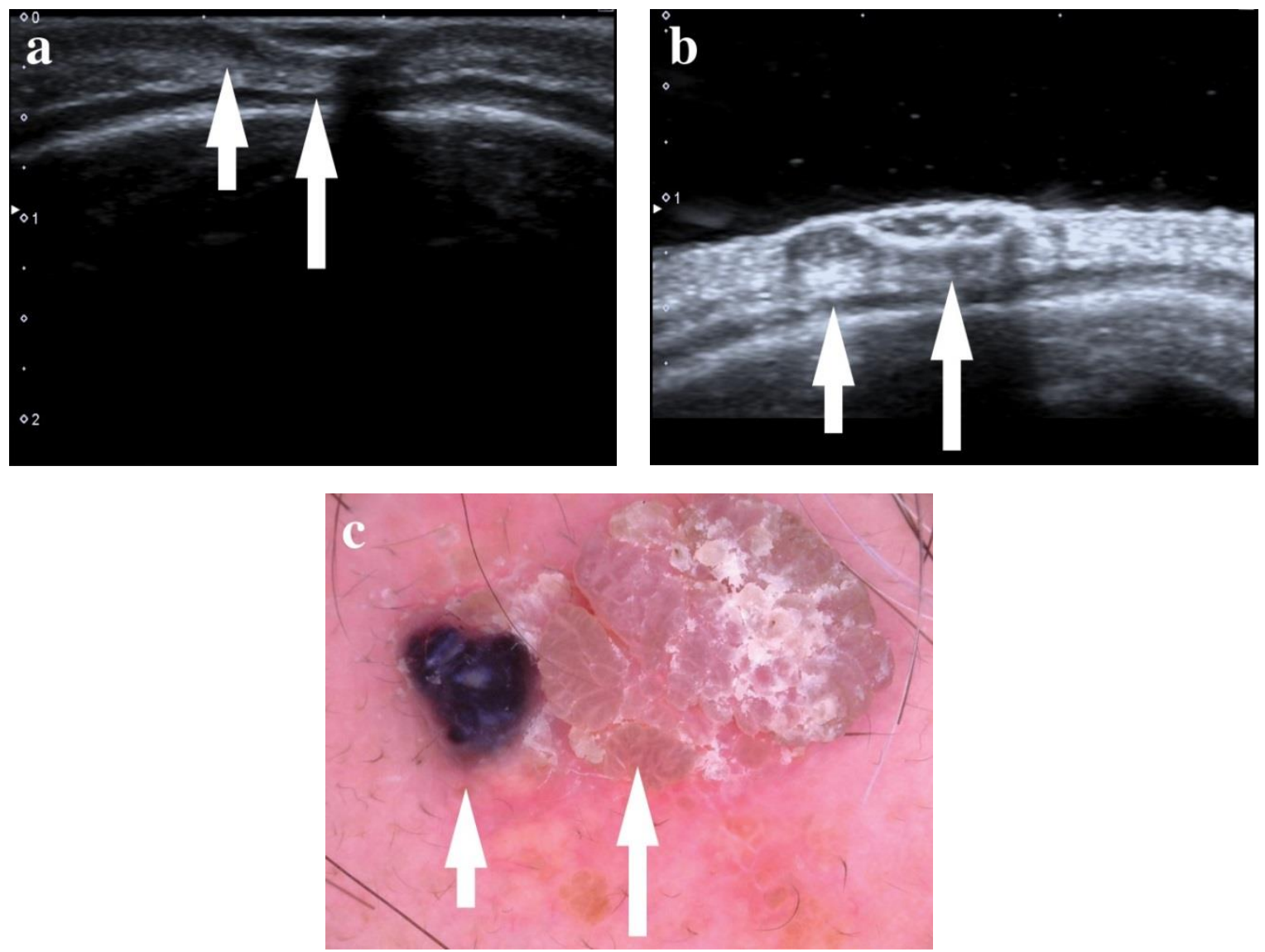

Figure 4. (a) Conventional ultrasound and (b) ultrasound with gel-pad of hemangioma with posterior enhancement (small white arrow) and keratosis with posterior shadowing (big white arrow). Ultrasound gel-pad provides an optimal possibility for revealing of different ultrasound acoustic phenomena. (c) Dermoscopy of the same cases: hemangioma (small black arrow) and keratosis (big black arrow) touching each other.

Nevus has a sharp and smooth mass margin (Table 1), hyperechoic or isoechoic inhomogeneous structure, and posterior enhancement as the retro acoustic phenomena. Following the nevus margins in many cases, we found surrounded hyperechoic rim (Figure 4b), which was very typical for this art of benign skin pathology. We noticed sharp together with smooth and oval shape margins in 33 of 35 benign pathology cases together, including naevi, keratoses, atheromas, and hemangiomas. The majority of melanomas and benign masses had hypoechoic structures. In half of the nevus cases, there were isoechoic structures and the L/T relation was 4.46 \pm 1.99 . Doppler analysis of the nevus defines absent of flow or low flow sometimes mainly peripheral, organized or disorganized, hyper-vascularity. Elastography stiffness is $0.78 \pm 0.45, \mathrm{P}<0.01$.

\section{Discussion}

We analyzed melanoma as malignant skin pathology and no other malignant skin diseases such as squamous cell carcinoma, basal cell carcinoma or lymphoma along with the aggressive nature of melanoma and the high rate of mortality, especially in their late diagnosis. For the differential diagnosis of melanoma with benign skin pathologies, included nevus, keratosis, hemangioma and atheroma, ultrasound multimodal criteria analysis gives profitable information. Depending on pathology a variety of criteria can occur. Due to the importance of the diagnostic, we have 
marked three main groups of criteria: A, B, and C: The most significant is the criteria collected in the group A, the criteria for the group B are less significant, and the criteria for the group $\mathrm{C}$ are least significant.

A. The criteria, which occur only at a current pathology;

B. The criteria, which always occur at a current pathology and as well can be seen at other pathologies;

C. The criteria, which not always occur at a current pathology as well as at other pathologies.

Group A: Hypoechoic structures with microcalcifications are specific for atheroma, while exclusively at melanomas multiple peduncles visualizations with help of Doppler were declared. Posterior shadow as the retro mass acoustic phenomena is common for the keratosis (Figure 4b). Several authors who studied lesions with crusting or keratinization, recommended to remove it, since they cause attenuation of the sound beam, reducing the accuracy of the test. Such an approach was helpful for the detailed examination of the mass structures but what we need is to use posterior shadowing as the diagnostic criteria for keratosis and not common for melanoma [15].

Group B: Hypoechoic echogenicity or absence of circular rim happened in all groups of patients with melanoma, but also in the small quantities in the benign pathology.

Group C: Sharp and jagged margins are common for melanoma. This type of margin changes can happen also in patients with a nevus. Hypervascularization of the mass in color Doppler (Figure 3) is common for melanoma and hemangioma.

For the differential diagnosis, it is necessary to find criteria that are specific or not specific for melanoma and benign pathology separately. The combination of different criteria can give the possibility to have the most realistic image impression. Evaluating our survey it becomes obvious that the most frequent and significant ultrasound criteria in malignant melanoma to be chosen are: sharp jagged well-defined margin, hypoechoic echogenicity, the multiple pedicle visualizations, the absence of the masses microcalcifications, anechoic structures and retro masses acoustic phenomena. Additional ultrasound criteria to be considered: homogenous internal echo pattern, central vascularization, disorganized vascularization, hypervascularization, multiple pedicels hyper vascularization also can occur in melanoma.

The L/T ratio of the malignant melanoma mass was higher $(7.9 \pm 1.96)$ than the ratio of benign pathologies (4.16 \pm 2.09$)$ with no significant statistical differences $(\mathrm{P}<0.5)$. Further studies are needed to prove some statistical nonsignificant data. Strain Elastography stiffness in the group of cutaneous melanoma stiffness was $2.95 \pm 0.18$, and in the benign skin pathologies, the average strain elastography stiffness was $0.96 \pm 0.59(\mathrm{P}<0.0001)$. To the author's knowledge, the first report concerning the additional use of a real-time tissue Elastography at the diagnosis of cutaneous melanoma was by two female patients with suspicious pigmented lesions on the back [20]. In contrast to the Elastography findings of both melanomas, an elastography examination of a nevus representing a "negative control" depicted a green pattern, representing a benign lesion. The strain ratio of the 29 malignant lesions ranged from 3.9 to 32.2 while by 38 benign lesions it ranged from 0.01 to 3.0. The maximum benign (3.0) and minimum malignant (3.9) strain ratios were defined without a priori reference criteria for such a differentiation. Instead, this ratio was established retrospectively. Although this study showed a $100 \%$ correlation between skin ultrasound Elastography and histology, the total number of patients was limited and the number of cases per pathological entity was relatively small [15]. Table 2 describes the ultrasound analytic criteria that are common for malignant melanoma and benign skin pathologies.

Table 2. Description of the common ultrasound analytic criteria for the melanoma and benign skin lesions

\begin{tabular}{|c|c|}
\hline Pathology & Description of ultrasound analysis \\
\hline Melanoma & $\begin{array}{l}\text { Sharp, well-defined margin, with absent of circular rim, hyperechoic mainly homogeneous, with absent of retro mass } \\
\text { acoustic phenomena. L/T relation is high (7.9). Doppler mass analysis mainly central, disorganized, } \\
\text { hypervascularization, with multiple pedicels. Elastography stiffness value is high in the melanoma (2.95). }\end{array}$ \\
\hline Atheroma & $\begin{array}{l}\text { Sharp and smooth margin with absent of the circular rim, hypoechoic, and mainly few echoic calcifications, with retro } \\
\text { mass acoustic phenomena - posterior enhancement. L/T relation is low (2.15). Doppler mass analysis shows absent or } \\
\text { aberrant flow. Elastography stiffness value is intermediate }(2.0) \text {. }\end{array}$ \\
\hline Hemangioma & $\begin{array}{l}\text { Sharp and smooth margin with absent of the circular rim, hypoechoic or hyperechoic mainly homogeneous, with absent } \\
\text { of retro mass acoustic phenomena and L/T relation is intermediate (4.17). Doppler mass analysis mainly central, } \\
\text { organized, hypervascularization, with single pedicels. Elastography stiffness is small (0.55). }\end{array}$ \\
\hline Keratosis & $\begin{array}{l}\text { Sharp and smooth margin with absent of the circular rim, hyperechoic mainly inhomogeneous retro mass acoustic } \\
\text { phenomena - posterior shadow. L/T relation is intermediate (4.76). Doppler mass analysis show absent of flow } \\
\text { sometimes single pedicels can be visualized and Elastography stiffness is small (1.21). }\end{array}$ \\
\hline Nevus & $\begin{array}{l}\text { Sharp and smooth mass margin with circular rim, hyperechoic or isoechoic inhomogeneous structure, and retro acoustic } \\
\text { phenomena - posterior enhancement. L/T relation is intermediate }(4.46) \text {. Doppler mass analysis shows absent or low } \\
\text { flow sometimes mainly peripheral or disorganized vascularization. Elastography stiffness is small }(0.78) \text {. }\end{array}$ \\
\hline
\end{tabular}




\section{Conclusion}

High-frequency ultrasound can take its place in the dermatology algorithm for differential diagnosis of malignant melanoma and benign skin lesions. The combination of certain ultrasound diagnostic analytic criteria, Doppler examination and elastography can help with the differential diagnosis of skin melanoma and several benign skin pathologies including atheromas, hemangiomas, keratoses, and naevi.

\section{Declaration of Competing Interest}

The authors declare that they have no known competing financial interests or personal relationships that could have appeared to influence the work reported in this paper.

\section{Ethical Approval}

All procedures performed in studies involving human participants were in accordance with the ethical standards of the institutional and/or national research committee and with the 1964 Helsinki declaration and its later amendments or comparable ethical standards.

\section{References}

[1] Rigel, D. S., Russak, J., \& Friedman, R. (2010). The Evolution of Melanoma Diagnosis: 25 Years Beyond the ABCDs. CA: A Cancer Journal for Clinicians, 60(5), 301-316. doi:10.3322/caac.20074.

[2] Leachman, S. A., Cassidy, P. B., Chen, S. C., Curiel, C., Geller, A., Gareau, D., ... Weinstock, M. A. (2015). Methods of Melanoma Detection. Cancer Treatment and Research, 51-105. doi:10.1007/978-3-319-22539-5_3.

[3] Van der Leest, R. J. T., de Vries, E., Bulliard, J.-L., Paoli, J., Peris, K., Stratigos, A. J., ... del Marmol, V. (2011). The Euromelanoma skin cancer prevention campaign in Europe: characteristics and results of 2009 and 2010. Journal of the European Academy of Dermatology and Venereology, 25(12), 1455-1465. doi:10.1111/j.1468-3083.2011.04228.x.

[4] Partsch, H. (1982). Measuring methods in dermatological angiology (author's transl). Zeitschrift fur Hautkrankheiten, 57(4), 227-246.

[5] Strasser, W., Vanscheidt, W., Hagedorn, M., \& Wokalek, H. (1986). B-scan ultrasound in dermatology. Fortschritte der Medizin, 104(25), 495.

[6] Wortsman, X. (2012). Common Applications of Dermatologic Sonography. Journal of Ultrasound in Medicine, 31(1), 97-111. doi:10.7863/jum.2012.31.1.97.

[7] Schroeder, R. J., Maeurer, J., Vogl, T. J., Hidajat, N., Hadijuana, J., Venz, S., ... \& Felix, R. (1999). D-galactose-based signalenhanced color Doppler sonography of breast tumors and tumorlike lesions. Investigative radiology, 34(2), 109-115.

[8] Marsden, J. R., Newton-Bishop, J. A., Burrows, L., Cook, M., Corrie, P. G., Cox, N. H., .. Walker, C. (2010). Revised U.K. guidelines for the management of cutaneous melanoma 2010. British Journal of Dermatology, 163(2), $238-256$. doi:10.1111/j.1365-2133.2010.09883.x.

[9] Fernández Canedo, I., de Troya Martín, M., Fúnez Liébana, R., Rivas Ruiz, F., Blanco Eguren, G., \& Blázquez Sánchez, N. (2013). Preoperative 15-MHz Ultrasound Assessment of Tumor Thickness in Malignant Melanoma. Actas DermoSifiliográficas (English Edition), 104(3), 227-231. doi:10.1016/j.adengl.2012.06.025.

[10] Lucas, V. S., Burk, R. S., Creehan, S., \& Grap, M. J. (2014). Utility of High-Frequency Ultrasound. Plastic Surgical Nursing, 34(1), 34-38. doi:10.1097/psn.0000000000000031.

[11] Botar Jid C, Bolboacă SD, Cosgarea R et al. (2015). Doppler ultrasound and strain elastography in the assessment of cutaneous melanoma: preliminary results. Medical Ultrasonography, 17(4): 509-514. doi:10.11152/mu.2013.2066.174.dus.

[12] Botar-Jid, C. M., Cosgarea, R., Bolboacă, S. D., Şenilă, S. C., Lenghel, L. M., Rogojan, L., \& Dudea, S. M. (2016). Assessment of Cutaneous Melanoma by Use of Very- High-Frequency Ultrasound and Real-Time Elastography. American Journal of Roentgenology, 206(4), 699-704. doi:10.2214/ajr.15.15182.

[13] Crișan, D., Badea, A. F., Crișan, M., Rastian, I., Solovastru, L. G., \& Badea, R. (2014). Integrative analysis of cutaneous skin tumours using ultrasonogaphic criteria. Preliminary results. Medical ultrasonography, 16(4), 285-290.

[14] Dasgeb, B., Morris, M. A., Mehregan, D., \& Siegel, E. L. (2015). Quantified ultrasound elastography in the assessment of cutaneous carcinoma. The British Journal of Radiology, 88(1054), 20150344. doi:10.1259/bjr.20150344.

[15] Barcaui, E. de O., Carvalho, A. C. P., Lopes, F. P. P. L., Piñeiro-Maceira, J., \& Barcaui, C. B. (2016). High frequency ultrasound with color Doppler in dermatology. Anais Brasileiros de Dermatologia, 91(3), 262-273. doi:10.1590/abd18064841.20164446 . 
[16] Leucht, W. (1996). Teaching atlas of breast ultrasound. Thieme Stuttgart.

[17] Marmur, E. S., Berkowitz, E. Z., Fuchs, B. S., Singer, G. K., \& Yoo, J. Y. (2010). Use of High-Frequency, High-Resolution Ultrasound Before Mohs Surgery. Dermatologic Surgery, 36(6), 841-847. doi:10.1111/j.1524-4725.2010.01558.x.

[18] Barcaui, E. de O., Carvalho, A. C. P., Valiante, P. M. N., \& Barcaui, C. B. (2014). High-frequency ultrasound associated with dermoscopy in pre-operative evaluation of basal cell carcinoma. Anais Brasileiros de Dermatologia, 89(5), 828-831. doi:10.1590/abd1806-4841.20143176.

[19] Olsen, L. O., Takiwaki, H., \& Serup, J. (1995). High-frequency ultrasound characterization of normal skin. Skin thickness and echographic density of 22 anatomical sites. Skin Research and Technology, 1(2), 74-80. doi:10.1111/j.16000846.1995.tb00021.x.

[20] Hinz, T., Wenzel, J., \& Schmid-Wendtner, M.-H. (2011). Real-time tissue elastography: A helpful tool in the diagnosis of cutaneous melanoma? Journal of the American Academy of Dermatology, 65(2), 424-426. doi:10.1016/j.jaad.2010.08.009. 